\title{
All the Related Therapeutic Methods of Traditional Chinese Medicine for Neck Pain and Low Back Pain
}

\author{
Qi-ling Yuan, Liang Liu and Yin-gang Zhang* \\ ${ }^{1}$ Department of Orthopaedics of the First Affiliated Hospital, Xi'an Jiaotong University, China
}

Submission: March 24, 2017, 2017; Published: May0 5, 2017

*Corresponding author: Yin-gang Zhang; Department of Orthopaedics of the First Affiliated Hospital, Medical School, Xi'an Jiaotong University, Xi'an 710061, China; Tel: +86-029-85323935; Email: zyingang@mail.xjtu.edu.cn

\begin{abstract}
Neck pain (NP) and low back pain (LBP) are common symptoms bothering people in daily life. There are many traditional Chinese medicine (TCM) therapeutic methods for NP and LBP in China, such as Chinese herbal medicine, acupuncture, moxibustion, tuina, chiropractic therapy, cupping, acupressure, Gua sha, acupotomy, Tai chi and Qigong. We reviewed and introduced all these methods above. Every method has its own specific curative efficacy; therefore these methods usually are used conjunctively. In the case of conventional western medicine treatment invalid, TCM may be a good choice.
\end{abstract}

Keywords: Neck pain; Low back pain; Traditional chinese medicine

Abbreviations: NP: Neck Pain; LBP: Low Back Pain; TCM: Traditional Chinese Medicine; CHM: Chinese Herbal Medicine

\section{Introduction}

Neck pain (NP) and low back pain (LBP) are common symptoms bothering people in daily life. In developed countries, more than $70 \%$ of people experience LBP, whereas approximately two-thirds of people experience NP at some point in their lifetimes [1]. Traditional Chinese medicine (TCM) has been used to treat various diseases in China and even throughout East Asia for more than 2000 years, and it still remains the choice for many people. Although acupuncture is a typical representative of TCM, it is only one of the various general therapies for NP and LBP, many other TCM therapeutic methods are still used extensively in China.

Chinese herbal medicine (CHM), which has been used for centuries in China, is generally formula-based, and single herbs are rarely used [2]. TCM holds that every medicinal substance has its strengths and its shortcomings, and each ingredient in the formula should be carefully balanced in quality and quantity, in order to accentuate its efficacy while reducing side effects. The herbs are not simply added in a cumulative fashion but are combined according to particular principles. First, through a unique diagnostic process, physicians discern the subtle patterns according to the symptoms of the individual, which then guides them to determine therapeutic strategies and to design or select the appropriate formulae. There are different ways of drug delivery. Firstly, CHM is usually administrated orally, and Duhuojisheng decoction is most frequently used
[3]. Secondly, it can be delivered by intravenous infusion, such as Mailuoning Injection [4]. Thirdly, it can also be used by external application, including topical CHM, CHM fumigation, and iontophoresis of $\mathrm{CHM}$, and these external administration methods can improve the drug concentration of diseased regions and reduce systemic side effects.

Acupuncture is a therapeutic method that prevents and cures disease by means of penetrating needles into specific acupoints on the human body, guided by the theory of meridians and acupoints of TCM [5]. There are various types of acupuncture styles, such as manual stimulation of the needles as well as electricity or heat stimulation of the needles. The therapeutic effects of acupuncture may have some favorable effects for NP and LBP [6]. Moxibustion is a TCM therapy which treats diseases by means of the heat generated by burning an herbal praeparatum mainly containing mugwort [7]. It is usually used in combination with acupuncture. Various methods of moxibustion therapy for neck or back pain have almost the same mechanisms, which may improve regional blood circulation, eliminate inflammatory reactions and the edema of nerve roots. Furthermore, the heat that follows mugwort burning may change the local microcirculation of the vertebra lumbalis or cervicalis. The acupoints on the body surface are activated and sensitized when individuals get sick. The areas of sensitivity, which are called heat-sensitive acupoints, are sensitive to thermal stimulus. These sensitive areas, on one hand, could reflect the pathological 
phenomena of diseases; on the other hand, they may be effective stimulus sites. Some studies showed that moxibustion was effective for NP or LBP [8]. As a part of TCM using manual therapy, tuina also emphasizes the meridians and acupoints as well as some anatomy and physiology [9].

Tuina is mainly composed of two components: soft tissue manipulation and backbone manipulation. The soft tissue techniques, which are analogous to massage, include stroking, kneading, and drumming. In contrast, the backbone manipulation is similar to mobilization and other regulation techniques. These techniques use manual operation procedures without thrust, which can maintain the range of the spine during physiological activity. Whereas, they also include procedures with thrust, which can make the spine exceed its physiological and anatomical range. Many papers published in Chinese had proved the favorable effects of Tuina.

Chiropractic therapy is an important part of TCM. In brief, according to the differences that exist in mechanisms and techniques, it can be classified into three approaches: rotation, traction or a combination [10]. Chiropractic therapy holds the principles: to reconstruct the balance of the musculoskeletal system through relaxing the muscles and rectifying abnormalities in the relationship between joints. Chiropractic therapy, which is based on the meridians and acupoints of TCM, differs from Western manipulation in theory and practice. It is mainly composed of two parts: relaxation of the soft tissues and bone setting. Some studies showed that chiropractic therapy was a good choice for NP or LBP [8]

Cupping is an ancient technique that aims to induce local congestion and blood stasis for healing. It works according to the principles of acupoints and meridians. The cups are made in different sizes and come in bamboo, plastic, glass or porcelain. The treatment consists of creating a vacuum by burning a taper inside a cup and quickly placing the cup over the selected area. TCM holds that the resulting suction effect invigorates blood and qi flows, unblocks the meridians, dispels dampness and cold, and eases swelling and pain. Cupping is effective for treating NP and LBP [8].

Acupressure uses the application of fingers and pressure to stimulate specific acupoints on the human body [11]. This noninvasive therapy was originally developed from TCM, which focuses on the balance of yin and yang and maintains the function of vital organs through circulation of blood and energy (chi) in the body. Acupressure was more effective than placebo and physical therapies for both NP and LBP [8]. Under the 0guidance of the basic theories of TCM, gua sha is a technique of scraping the surface of body at targeted locations using specific scraping instruments to prevent disease [12]. After scraping, ecchymosis and petechiae are distinct at the local skin site, which are called "sha"; it is believed that poisons in the inner body have been extracted in the shape of the sha [12].
Gua shais a technique used for pain relief, muscular tension, and spasms. Its main mechanisms are, on one hand, to strengthen regional circulation and elevate the temperature of local tissue and, on the other hand, to boost the pain threshold of regional tissue. Gua sha is effective for NP and LBP [8].

Acupotomy is a combined simple therapy of TCM and modern surgical principles which is used as the main tool for treating chronic soft tissue injury and bone hyperplasia with a bladed needle that has a thick flat-head and a cylindrical body. The aim of acupotomy is to recover the kinetic state of soft tissue from peeling adhesion, remove attached tissues, and reduce pressure on the nerve. Acupotomy has many benefits because it converts open surgery to closed surgery, thus reducing risk, time, and cost. This method leaves only a small scar that will fade with time. Some studies showed that acupotomy was effective for LBP [13].

Tai chi is the name for an immemorial Chinese philosophy and conveys the ideas of the supreme, extreme, absolute, and unique [14]. Tai chi quan is a perfect combination of Chinese national dialectical theoretical thinking and martial arts, arts, guiding methodology, and TCM [14]. Tai chi as a high-level expression of the human body has multiple functions, which include taking care of one's temperament, building one's body, and the art of attack and defense. Two studies showed the Taichi may be effective for LBP [8].

Qigong [15], a therapeutic Chinese practice that has been used for a thousand years to guard against and treat disease, refers to a mind- and body-training method that focuses on breathing adjustment, physical activity modulation, and willing adjustment. Qigong is part of TCM and follows the principles of regulating "qi" (energy), which is assumed to harmonize mind and body so as to reduce pain. Five-animal exercise [15] is a subgroup of qigong, which imitates the movements and expressions of five animals and was an important regimen in ancient times. The effects of Qigong for NP have be showed favorable in some studies [8].

\section{Conclusion}

There are many TCM therapeutic methods for NP and LBP, and every method has its own specific curative efficacy, therefore these methods usually are used conjunctively. In the case of conventional western medicine treatment invalid, TCM may be a good choice.

\section{Acknowledgement}

The authors are grateful for the support of the Natural Scientific Fund of China (no. 81371987).

\section{References}

1. Andersson G (1997) The epidemiology of spinal disorders. The adult spine. Principles and practice.

2. Li CP (2003) Chinese herbal medicine: Book Tree. USA. 
3. Ma Y, Cui J, Huang M, Meng K, Zhao Y (2013) Effects of Duhuojisheng Tang and combined therapies on prolapse of lumbar intervertebral disc: a systematic review of randomized control trails. J Tradit Chin Med 33(2): 145-155.

4. Zhi MX, Zhang GB, Hou JC, Yang YH, Wan ZZ (2009) Peridural injection of Mailuoning compound liquor for treatment of prolapse of lumbar intervertebral disc in 100 cases. J Tradit Chin Med 29(1): 6-8.

5. Stux G, Berman B, Pomeranz B (2003) Basics of acupuncture. Springer.

6. Yuan QL, Wang P, Liu L, Sun F, Cai YS, et al. (2016) Acupuncture for musculoskeletal pain: A meta-analysis and meta-regression of shamcontrolled randomized clinical trials. Sci Rep 6: 30675.

7. Chiu ML, Li L (1993) Chinese acupuncture and moxibustion: Churchill Livingstone Edinburgh etc. Churchill Livingstone, England.

8. Yuan QL, Guo TM, Liu L, Sun F, Zhang YG (2015) Traditional Chinese medicine for neck pain and low back pain: a systematic review and meta-analysis. PLoS One 10: e0117146.

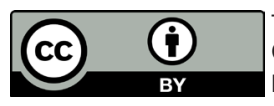

This work is licensed under Creative Commons Attribution 4.0 Licens

DOI: 10.19080/JCMAH.2017.02.555582
9. Yan JT (2003) Tuina Science. Beijing: China Press of Traditional Chinese Medicine, China.

10. Zhao GH, Ji Q (2008) Progress in manual therapy for cervical spondylotic radiculopathy. Beijing J Tradit Chin Med J 27: 817.

11. McFadden KL, Hernández TD (2010) Cardiovascular benefits of acupressure (Jin Shin) following stroke. Complementary therapies in medicine 18(1): 42-48.

12. Nielsen A (2012) Gua Sha: a traditional technique for modern practice. Churchill Livingstone, England.

13. Kim HJ, Jeon JH, Kim YI (2016) Clinical Effect of Acupotomy Combined with Korean Medicine: A Case Series of a Herniated Intervertebral Disc. J Acupunct Meridian Stud 9(1): 31-41.

14. Lan C, Lai JS, Chen SY (2002) Tai Chi Chuan. Sports Medicine 32: 217224.

15. Koh T (1982) Qigong-Chinese breathing exercise. The American journal of Chinese medicine 10(1-4): 86-91.

\section{Your next submission with Juniper Publishers will reach you the below assets}

- Quality Editorial service

- Swift Peer Review

- Reprints availability

- E-prints Service

- Manuscript Podcast for convenient understanding

- Global attainment for your research

- Manuscript accessibility in different formats

( Pdf, E-pub, Full Text, Audio)

- Unceasing customer service

Track the below URL for one-step submission

https://juniperpublishers.com/online-submission.php 